\title{
ARTICLE
}

Epidemiology

\section{Risk of diabetes after para-aortic radiation for testicular cancer}

Harmke J. Groot ${ }^{1}$, Jourik A. Gietema ${ }^{2}$, Berthe M. P. Aleman ${ }^{3}$, Luca Incrocci ${ }^{4}$, Ronald de Wit ${ }^{5}$, J. Alfred Witjes ${ }^{6}$, Gerard Groenewegen ${ }^{7}$, Peter de Brouwer ${ }^{8}$, Otto W. M. Meijer ${ }^{9}$, Maarten C. C. M. Hulshof ${ }^{10}$, Hetty A. van den Berg ${ }^{11}$, Tineke J. Smilde ${ }^{12}$, Ben G. L. Vanneste ${ }^{13}$, Maureen J. Aarts ${ }^{14}$, Alphonsus C. M. van den Bergh ${ }^{15}$, J. Martijn Kerst ${ }^{16}$, Alexandra W. van den Belt-Dusebout ${ }^{1}$, Sjoukje Lubberts $^{17}$, Katarzina Jóźwiak $^{18}$, Simon Horenblas ${ }^{19}$, Flora E. van Leeuwen ${ }^{1}$ and Michael Schaapveld ${ }^{1}$

BACKGROUND: While the risk of diabetes is increased following radiation exposure to the pancreas among childhood cancer survivors, its association among testicular cancer (TC) survivors has not been investigated.

METHODS: Diabetes risk was studied in 2998 1-year TC survivors treated before 50 years of age with orchidectomy with/without radiotherapy between 1976 and 2007. Diabetes incidence was compared with general population rates. Treatment-specific risk of diabetes was assessed using a case-cohort design.

RESULTS: With a median follow-up of 13.4 years, 161 TC survivors were diagnosed with diabetes. Diabetes risk was not increased compared to general population rates (standardised incidence ratios (SIR): 0.9; 95\% confidence interval (95\% Cl): 0.7-1.1). Adjusted for age, para-aortic radiotherapy was associated with a 1.66-fold (95\% Cl: 1.05-2.62) increased diabetes risk compared to no radiotherapy. The excess hazard increased with 0.31 with every $10 \mathrm{~Gy}$ increase in the prescribed radiation dose $(95 \% \mathrm{Cl}: 0.11-0.51$, $P=0.003$, adjusted for age and $\mathrm{BMI})$; restricted to irradiated patients the excess hazard increased with 0.33 (95\% $\mathrm{Cl}$ : -0.14 to 0.81 , $P=0.169)$ with every $10 \mathrm{~Gy}$ increase in radiation dose.

CONCLUSION: Compared to surgery only, para-aortic irradiation is associated with increased diabetes risk among TC survivors.

British Journal of Cancer (2018) 119:901-907; https://doi.org/10.1038/s41416-018-0248-x

\section{INTRODUCTION}

Several studies among childhood cancer and Hodgkin lymphoma survivors have reported that infradiaphragmatic radiotherapy may increase risk of diabetes. ${ }^{1-4}$ A recent study by van Nimwegen and colleagues showed that Hodgkin lymphoma survivors treated with para-aortic irradiation at doses of $\geq 36$ Gray had a 1.8-fold increased risk of diabetes compared to patients without radiotherapy. ${ }^{4}$ Lower doses, which are more common in testicular cancer (TC) treatment, did not significantly increase diabetes risk in this study.

Although para-aortic radiotherapy (PAO-RT) is no longer used for treatment of non-seminoma patients nowadays and only rarely for treatment of stage I seminoma patients, it has long been part of TC treatment. PAO-RT for TC will generally result in irradiation of the head and body of the pancreas, which contains part of the insulin-producing beta-cells. ${ }^{5}$ Only one previous study assessed diabetes prevalence after radiotherapy for testicular cancer. Haugnes et al. observed a diabetes prevalence of $10.2 \%$ among patients treated with radiotherapy in 1980-1994 and screened for (risk factors for) cardiovascular disease (CVD) in 2008, which was 2.3-fold higher compared to prevalence among general population controls. ${ }^{6}$ The effect of radiation dose on diabetes risk was not assessed in this study. It is important to ascertain whether radiotherapy is associated with increased diabetes risk among TC survivors, since diabetes is an important predictor of premature mortality ${ }^{7}$ and may increase risk of coronary artery disease. ${ }^{8,9}$ Establishing a dose-response relationship is clinically important since radiation doses to organs at risk can be significantly reduced with modern radiation techniques and dose constraints to the pancreas can be introduced in radiation planning for TC and other

\footnotetext{
${ }^{1}$ Department of Epidemiology, Netherlands Cancer Institute, Plesmanlaan 121, 1066 CX Amsterdam, The Netherlands; ${ }^{2}$ Department of Medical Oncology, University Medical Center Groningen, Hanzeplein 1, 9713 GZ Groningen, The Netherlands; ${ }^{3}$ Department of Radiation Oncology, Netherlands Cancer Institute, Plesmanlaan 121,1066 CX Amsterdam, The Netherlands; ${ }^{4}$ Department of Radiation Oncology, Erasmus Medical Center Cancer Institute, 's-Gravendijkwal 230 , 3015 CE Rotterdam, The Netherlands; ${ }^{5}$ Department of Medical Oncology, Erasmus Medical Center Cancer Institute, 's-Gravendijkwal 230, 3015 CE Rotterdam, The Netherlands; ${ }^{6}$ Department of Urology, Radboud University Medical Center, Geert Grooteplein Zuid 10, 6525 GA Nijmegen, The Netherlands; ${ }^{7}$ Department of Medical Oncology, University Medical Center Utrecht, Heidelberglaan 100,3584 CX Utrecht, The Netherlands; ${ }^{8}$ Department of Radiation Oncology, Dr. Bernard Verbeeten Institute, Brugstraat 10, 5042 SB Tilburg, The Netherlands; ${ }^{9}$ Department of Radiation Oncology, VU University Medical Center Amsterdam, De Boelelaan 1117, 1081 HV Amsterdam, The Netherlands; ${ }^{10}$ Department of Radiation Oncology, Amsterdam Medical Center, Meibergdreef 9, 1105 AZ Amsterdam, The Netherlands; ${ }^{11}$ Department of Radiotherapy, Catharina Hospital, Michelangelolaan 2 , 5623 EJ Eindhoven Eindhoven, The Netherlands; ${ }^{12}$ Department of Medical Oncology, Jeroen Bosch Hospital, Den Bosch, Henri Dunantstraat 1, 5223 GZ 's-Hertogenbosch, The Netherlands; ${ }^{13}$ Department of Radiotherapy, MAASTRO-clinic, Dr. Tanslaan 12, 6229 ET Maastricht, The Netherlands; ${ }^{14}$ Department of Medical Oncology, Maastricht University Medical Centre, P. Debyelaan 25, 6229 HX Maastricht, The Netherlands; ${ }^{15}$ Department of Radiation Oncology, University Medical Center Groningen, Hanzeplein 1, 9713 GZ Groningen, The Netherlands; ${ }^{16}$ Department of Medical Oncology, Netherlands Cancer Institute, Plesmanlaan 121, 1066 CX Amsterdam, The Netherlands; ${ }^{17}$ Department of Medical Oncology, University

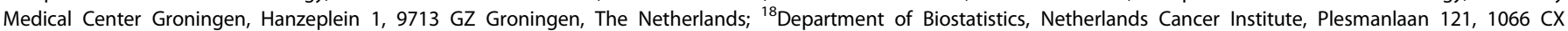
Amsterdam, The Netherlands and ${ }^{19}$ Department of Urology, Netherlands Cancer Institute, Plesmanlaan 121, 1066 CX Amsterdam, The Netherlands Correspondence: Michael Schaapveld (m.schaapveld@nki.nl)
}

Received: 9 January 2018 Revised: 19 July 2018 Accepted: 2 August 2018 Published online: 9 October 2018 
malignancies. Therefore, this study aims to assess the association of radiation dose and diabetes risk after PAO-RT in a large multicenter cohort of TC survivors.

\section{METHODS}

Study population and design

A hospital-based cohort was established including 6312 TC patients who were treated from January 1976 to December 2007 in 13 Dutch hospitals and were younger than 50 years at TC diagnosis. This cohort included 1874 5-year TC survivors treated between 1976 and 1995, entered in previous studies on cardiovascular disease (CVD) risk among TC survivors. ${ }^{10,11}$ Patients were identified through tumour registries and through the Netherlands Cancer Institute. Details on patient selection are presented in Supplemental Figure S1. For all patients in our hospital-based cohort, basic tumour and primary treatment characteristics were available.

In the current study, we aim to assess risk of diabetes among patients treated with radiotherapy as part of their primary treatment compared to risk of diabetes among patients treated with surgery only. Therefore, we first selected all patients from the hospital-based cohort who received either surgery only or surgery with radiotherapy as part of primary treatment and were aged between 12 and 50 years at orchidectomy and were alive 1 year after TC diagnosis $(N=3746$, Supplemental figure S1). For $68.6 \%$ of the cohort $(N=2568)$ and for $76.7 \%$ of the subcohort $(N=544)$, diabetes and cardiovascular disease follow-up was available through the GP and/or medical files until at least January 2011. We excluded all patients without any cardiovascular disease follow-up (575 patients); those who died or were lost to follow-up within 1 year (71 patients), those who received chemotherapy in the first year after TC diagnosis (85 patients) and who developed diabetes before TC (1 patient) or within 1 year after TC diagnosis (16 patients). This left 2998 patients for analysis. We subsequently identified all patients who had developed diabetes in our study cohort $(N=161$, Supplemental figure S1).

Similarly, we selected all patients from the subcohort who had received either surgery only or surgery with radiotherapy as primary treatment and who had survived at least 1 year after TC diagnosis $(N=614)$.

We used a case-cohort design to facilitate detailed treatment data collection while allowing assessment of multiple treatmentassociated outcomes. ${ }^{12} \mathrm{~A}$ hospital-stratified subcohort comprising $15 \%$ of the base cohort ( $25 \%$ in the two coordinating hospitals) was randomly selected, without taking cardiovascular or oncological follow-up into account. This subcohort comprised 1175 TC patients. For all randomly sampled patients in the subcohort and for all patients who developed diabetes (which includes patients who developed diabetes and were sampled in the subcohort), detailed treatment data were abstracted from the medical charts, including chemotherapy regimens, number of cycles and cumulative doses, radiotherapy fields and doses for primary treatment as well as relapse treatment. The study protocol was submitted to the Institutional Review Board of the Netherlands Cancer Institute, which waived the requirement for individual patient consent.

\section{Treatment}

All patients underwent orchidectomy. Radiotherapy for stage I and II seminomas typically comprised irradiation of the infradiaphragmatic para-aortic, ipsilateral, iliacal and inguinal lymph nodes. Irradiation of the para-aortic and iliac lymph nodes constitutes the dogleg radiation field. ${ }^{11,13}$ For left-sided tumours the para-aortic fields were usually extended to include the left renal hilar nodes. The radiation dose used in the treatment of seminoma patients gradually declined over time from 30 to 35 Gray in the 1980s to 26 Gray from 1990 onwards. ${ }^{10,14}$ Since the mid-2000s, stage I seminoma patients are increasingly treated with either surveillance, or in case of at least two risk factors, 20 Gray radiotherapy or 1 cycle of carboplatin (AUC 7). ${ }^{15-17}$ Before 1985, treatment for stage I or IIA non-seminoma frequently included adjuvant radiotherapy with a dose of 40-50 Gray in 20-25 fractions. ${ }^{13,14}$ From 1985 onwards, stage I non-seminoma patients were generally treated under watchful waiting protocols, depending on disease severity and prognostic factors. ${ }^{13,17}$

\section{Outcome assessment}

Information on date of diagnosis, type of and treatment for diabetes, was obtained between 2013 and 2016 from the medical records and/or questionnaires sent to the patient's general practitioner (GP) and from medical correspondence with the treating physician. In Dutch guidelines for general practitioners, diabetes is typically defined as fasting plasma glucose $\geq 7.0 \mathrm{mmol} / /{ }^{18}$ Population screening for diabetes is not part of current Dutch GP guidelines, hence, diabetes case ascertainment was based on clinical presentation to regular care (see Supplement S2).

\section{Statistical analysis}

Time at risk started 1 year after start of TC treatment and ended at date of diabetes diagnosis, date of death or date of last follow-up, whichever occurred first. Patients who relapsed were censored at the date of relapse if treatment for the relapse included chemotherapy and relapse treatment was started 1 year or more after TC diagnosis. The last medical follow-up as recorded in the medical files was used when no follow-up information from GP questionnaires was available. In the cohort, follow-up for diabetes and other cardiovascular disease risk factors was derived from information from the medical record and the GP in $32.2 \%$, from the GP only in $61.4 \%$ and from the medical record only in $6.4 \%$ of the patients; in the subcohort these percentages were $81.6 \%, 2.9 \%$ and $15.5 \%$, respectively. In total, $11.2 \%$ of the patients with diabetes in the subcohort (four patients) were identified through information from the medical record only.

Comparison with the general population. Incidence of diabetes was compared with sex-, age- (5-year strata), and calendar yearspecific diabetes incidence rates in the Dutch population. Diabetes incidence rates for the Dutch population were obtained from electronic health records of general practitioners participating in NIVEL Primary Care Database and were available for 2002-2016 (Number NZR-00316.030). Therefore, analyses were left censored at January 1, 2002. For more details on diabetes population, reference rates and calculation of standardised incidence ratios we refer to Supplemental file S3.

Case-cohort comparisons: assessing the relationship between treatment and diabetes risk. Cumulative incidence of diabetes was estimated in the presence of death as competing risk, and trends over time were evaluated using competing risk regression models with adjustment for age. ${ }^{19}$ Missing information on covariates were imputed using chained equations (MICE), ignoring patient clusters, and creating 20 datasets $^{18,19}$. Disease stage, radiation field and smoking were imputed with multinomial logistic regression, while weight, height and radiation dose were imputed using linear regression. In all imputation models, year and age at treatment, histology, age, hospital of diagnosis, diabetes status and cumulative hazards of diabetes calculated with a Cox regression model before imputation, were included as extra covariates. Associations of TC treatment with diabetes risk were assessed using multivariable Cox regression models with time since TC treatment as time scale. Barlow's inverse probability weights were used to adjust the partial likelihood function for our case-cohort design $^{12}$. TC treatment was entered in the model as a time-varying variable. Effects of treatment were evaluated accounting for the effects of other covariates where appropriate. Modification of a radiation-associated diabetes risk was assessed by inclusion of 


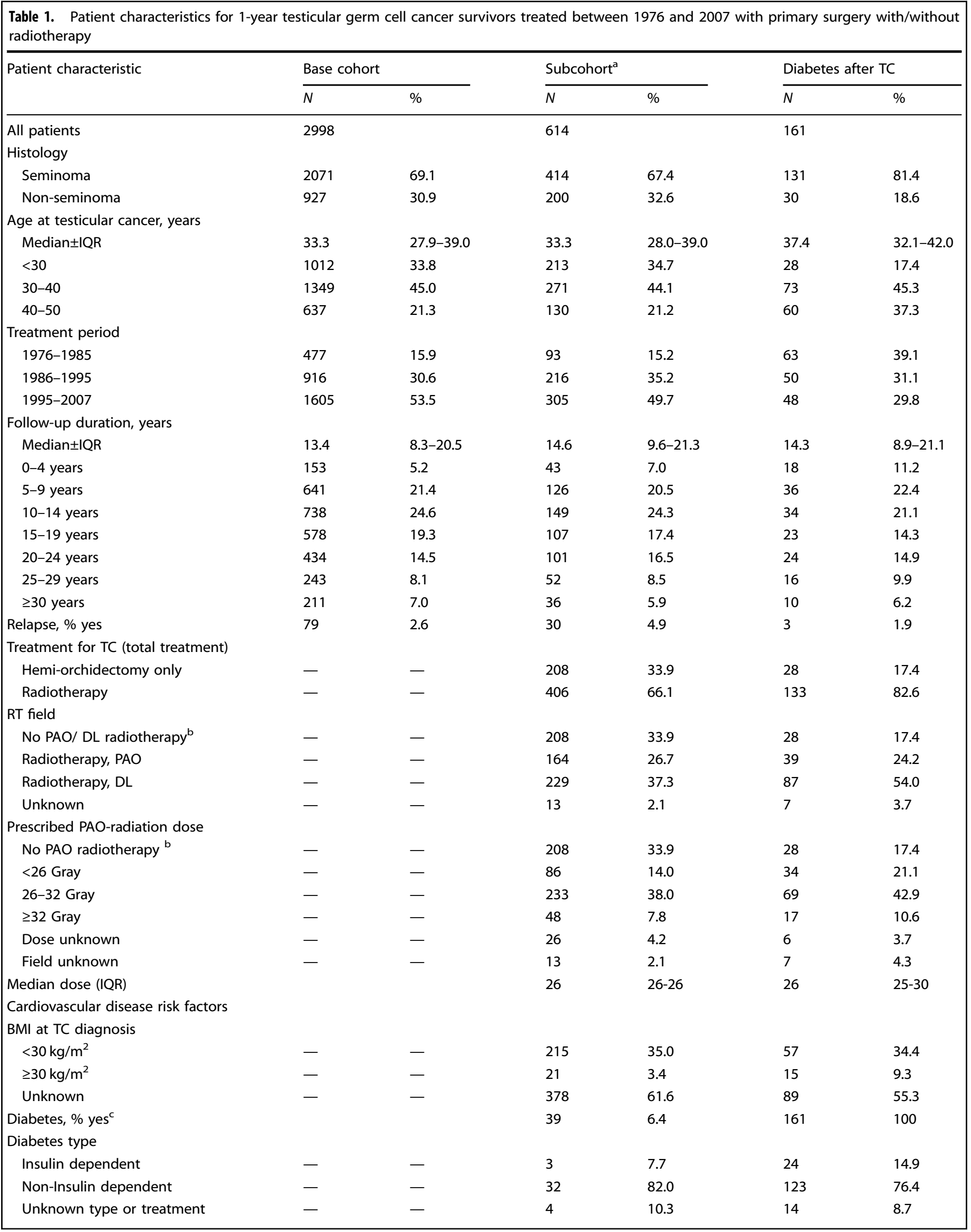




\begin{tabular}{|c|c|c|c|c|c|c|}
\hline \multirow[t]{2}{*}{ Patient characteristic } & \multicolumn{2}{|c|}{ Base cohort } & \multicolumn{2}{|c|}{ Subcohort $^{a}$} & \multicolumn{2}{|c|}{ Diabetes after TC } \\
\hline & $N$ & $\%$ & $N$ & $\%$ & $N$ & $\%$ \\
\hline \multicolumn{7}{|c|}{ Hypercholesterolemia at TC diagnosis } \\
\hline Yes & - & - & 5 & 0.8 & 3 & 1.9 \\
\hline No & - & - & 574 & 93.5 & 141 & 87.6 \\
\hline Yes & - & - & 10 & 1.6 & 9 & 5.6 \\
\hline No & - & - & 568 & 92.5 & 136 & 84.5 \\
\hline Unknown & - & - & 36 & 5.9 & 16 & 9.9 \\
\hline \multicolumn{7}{|c|}{ Smoking status at TC diagnosis } \\
\hline Yes & - & - & 181 & 29.5 & 55 & 34.2 \\
\hline \multicolumn{7}{|c|}{ Vital status at date of last contact } \\
\hline Alive & 2884 & 95.6 & 587 & 95.6 & 135 & 83.9 \\
\hline Died & 97 & 3.2 & 22 & 3.6 & 26 & 16.2 \\
\hline Emigrated & 37 & 1.2 & 5 & 0.8 & 0 & 0 \\
\hline \multicolumn{7}{|l|}{ Age at end of follow-up } \\
\hline Median $\pm I Q R$ & 48.2 & $40.9-55.7$ & 47.4 & $39.7-54.7$ & 51.9 & $45.1-59.2$ \\
\hline
\end{tabular}

interaction terms for age, obesity $\left(\mathrm{BMI}>30 \mathrm{~kg} / \mathrm{m}^{2}\right.$ ) and smoking with radiotherapy. To assess an excess relative risk for prescribed radiation dose in relation to diabetes risk in our case-cohort setting, the linear increase in the HRs over categories of prescribed dose was estimated separately for each imputed data set using weighted least square regression with weights equal to 1/variance of each HR and subsequently averaged over all 20 data sets. Cox regression model estimates from the imputed data sets were pooled using Rubin's rule. ${ }^{20}$ Dose categories were chosen such that each category represented at least five diabetes cases $(0, \leq 24$, $25,26,27-30,31-35,36-40$ and $\geq 40$ Gray) and confidence intervals of the HRs were based on Wald test. The proportional hazards assumption was assessed using residual-based methods. STATA (version 13.1, Statacorp LP, College Station, TX) statistical software was used for analysis. A $P \leq 0.05$ was considered significant.

\section{RESULTS}

Patient characteristics

Our study cohort comprised 2071 (69.1\%) seminoma and 927 nonseminoma TC patients (30.9\%). Median age at TC treatment was 33.3 years (Interquartile range (IQR): 27.9-39.0 years). The median follow-up was 13.4 years, during which 161 TC patients $(5.3 \%)$ developed diabetes. For $29.6 \%$ of the TC patients, follow-up exceeded 20 years. Among patients with diabetes, the median interval until diabetes was 14.3 years (IQR: 8.9-21.1 years). The median age at TC treatment was 37.4 years (IQR: $32.1-42.0$ years) for patients with diabetes; the median age at diabetes diagnosis was 51.9 years (IQR: $45.1-59.2$ years).

Of all patients in the subcohort, comprising 414 seminoma $(67.4 \%)$ and 200 non-seminoma patients (32.6\%), 406 patients $(66.1 \%)$ had received radiotherapy (Table 1$)$. Median follow-up was 15.8 years (IQR: 8.4-23.4 years) for patients treated with surgery only and 11.8 years (IQR: 6.2-19.8 years) for patients treated with radiotherapy. Of the seminoma patients in the subcohort, $6.3 \%$ underwent only surgery. The median radiotherapy dose to the para-aortic field was 30 Gray (range: 16-70 Gray) in 1976-1985 and decreased to 26 Gray (range: 17-51 Gray) during 1986-1995 (Supplemental table S4 and supplemental figure S5). Of the nonseminoma patients treated before 1985, 31.9\% received infradiaphragmatic radiotherapy with a median dose of 40 Gray (range: 30-70).

Diabetes risk compared to the general population: case-cohort analysis

Diabetes risk among TC patients did not differ from the diabetes risk expected based on incidence rates for the general Dutch male population (standardised incidence rate (SIR): 0.9, 95\% Cl: 0.7-1.1, Supplemental table S6). The SIR for diabetes was not increased for patients treated with radiotherapy (SIR: 1.0, 95\% Cl: 0.8-1.2) and diabetes risk did not differ by age at TC diagnosis ( $P$ heterogeneity: 0.970). SIRs were similar in the full cohort analysis, although risk was lower than expected for non-irradiated patients (SIR: 0.4, 95\% Cl: 0.3-0.6).

Diabetes risk after infradiaphragmatic radiotherapy: case-cohort analyses

The cumulative incidence of diabetes was $6.1 \%$ (95\% Cl: $5.0-7.4 \%)$ at 20 years and $15.6 \%$ (95\% Cl: $12.4-19.2 \%)$ at 30 years after TC treatment. Adjusted for age at TC diagnosis patients treated with para-aortic radiotherapy had a significantly higher risk of diabetes than patients who underwent only surgery $(P<0.001$; Fig. 1$)$. Patients with a prescribed radiation dose of $>26$ Gray to a paraaortic field had a 30-year cumulative incidence of $16.7 \%(95 \% \mathrm{Cl}$ : $11.9-22.2 \%)$, compared to a 30-year cumulative incidence of $9.5 \%$ $(95 \% \mathrm{Cl}: 5.0-15.7 \%)$ for patients treated with surgery only ( $P$ value: 0.122 , adjusted for age). 


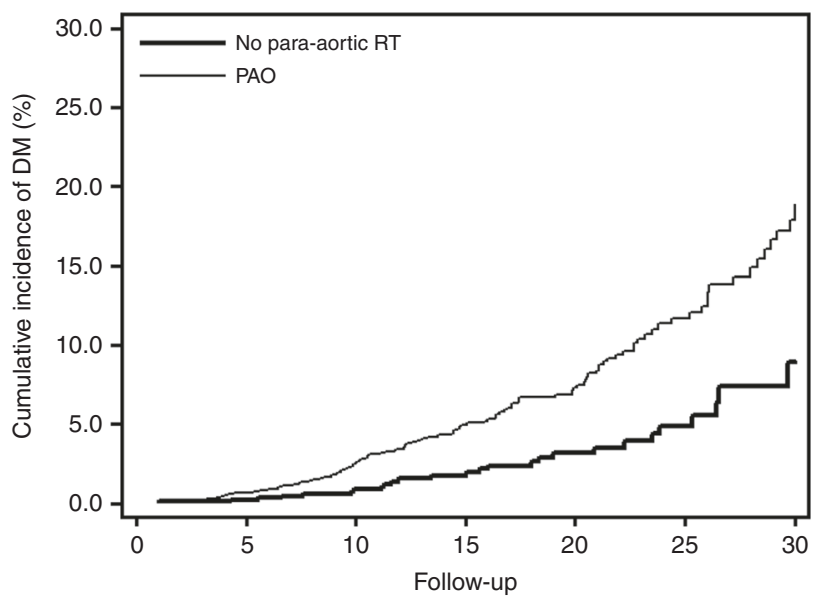

Fig. 1 Incidence of diabetes among testicular cancer survivors treated with para-aortic radiotherapy compared to orchidectomy only. PAO para-aortic irradiation. Diabetes incidence was significantly increased after para-aortic radiotherapy $(P<0.001)$
Adjusted for age, para-aortic RT was associated with a 1.66-fold (95\% Cl: 1.05-2.62; Table 2) increased diabetes risk compared to no radiotherapy. When adjusting for $\mathrm{BMI}$ and age (as continuous variables), risk decreased to 1.54 (95\% Cl: 0.96-2.45). A radiation dose of $\leq 26$ Gray to the para-aortic field was associated with a 1.29-fold (95\% Cl: 0.79-2.11) increased diabetes risk while doses of 27-32 Gray and $\geq 33$ Gray were associated with a 2.26 -fold $(95 \% \mathrm{Cl}$ : 1.22-4.21) and a 1.94-fold (95\% Cl: 0.95-3.97) increased diabetes risk, respectively (P-trend: 0.011). The excess hazard increased with 0.31 with every 10 Gray increase in the prescribed radiation dose (95\% Cl: $0.11-0.51, P=0.003$, Fig. 2). The excess risk among irradiated patients increased with $0.33(95 \% \mathrm{Cl}:-0.14$ to $0.81, P=$ 0.169 ) with every $10 \mathrm{~Gy}$ increase in radiation dose.

Obesity at TC diagnosis was an independent risk factor for diabetes, associated with a 2.52 -fold (95\% Cl: $1.31-4.87)$ increased diabetes risk, but did not modify the association between radiotherapy and diabetes risk $(P$-interaction $=0.496)$. The effect of infradiaphragmatic radiotherapy on diabetes risk was also not modified by smoking or age. Complete case analyses, which only included patients who had no missing data on radiation fields and dose, provided similar results (Supplemental table S7).

Table 2. Multivariable analysis for diabetes risk by treatment among TC survivors (case-cohort study with detailed treatment data)

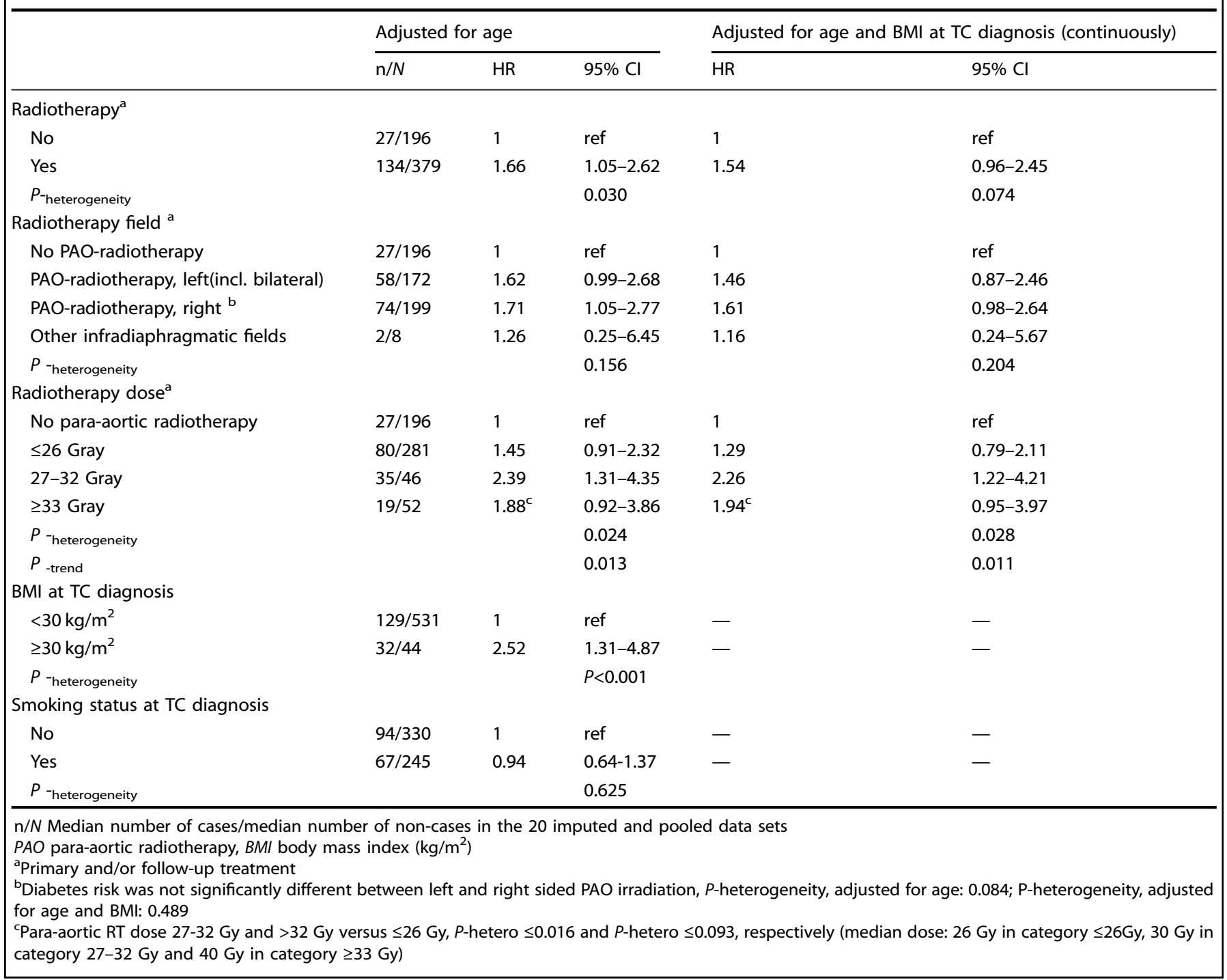




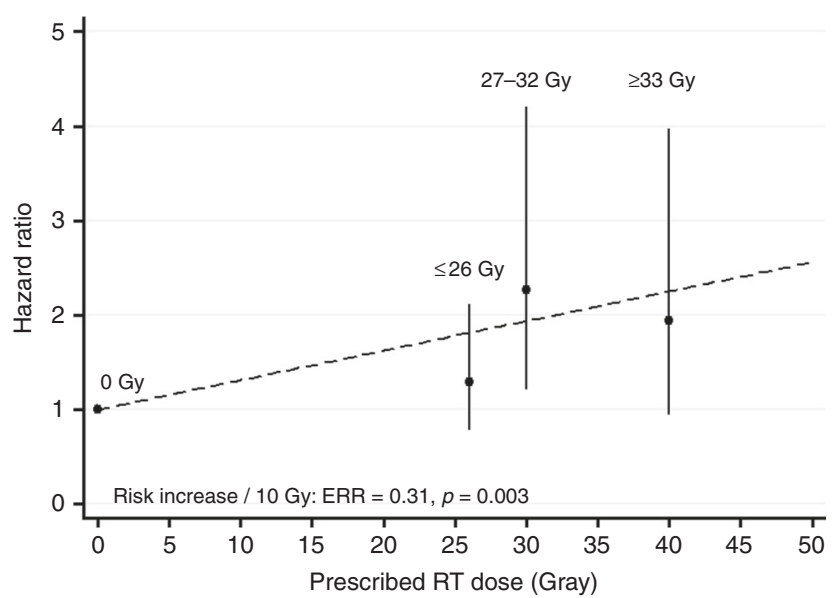

Fig. 2 Risk of diabetes by prescribed para-aortic radiation dose among testicular cancer survivors. HRs for diabetes risk for prescribed dose categories are plotted at the mean dose within each category ( $26 \mathrm{~Gy}$ for $\leq 26 \mathrm{GY}, 30 \mathrm{~Gy}$ for $27-32 \mathrm{~Gy}$ and $40 \mathrm{~Gy}$ for $\geq 33 \mathrm{~Gy}$ ). Vertical lines represent the $95 \% \mathrm{Cl}$ for each category of dose. The HRs for determining the excess risk increase per 10 Gray (ERR) were derived from a model with adjustment for age and BMI continuously. The excess risk was estimated based on the following dose categories: 10-24 Gy, $25 \mathrm{~Gy}, 26 \mathrm{~Gy}, 27-30 \mathrm{~Gy}, 31-39 \mathrm{~Gy}$ and $\geq 40$ Gy, with median doses of 21, 25, 26, 30, 33, 40 and 50 Gy

\section{DISCUSSION}

In this large cohort study we observed a 1.66 -fold increased risk of diabetes among TC survivors treated with para-aortic radiotherapy compared to TC survivors treated with surgery only, adjusted for age. Diabetes risk increased with a higher para-aortic radiation dose. However, diabetes risk after exposure to radiation was not increased compared to that of males in the Dutch general population, while diabetes risk was lower than expected among survivors treated with surgery only.

Four studies previously investigated diabetes risk after paraaortic radiotherapy in cancer survivors. ${ }^{1,2,4}$ In a French-British cohort, de Vathaire and colleagues found an 11-fold increased diabetes risk among childhood cancer survivors irradiated with 20 Gray to the pancreatic tail compared to those not treated with radiotherapy; risk increased with increasing dose up to $\geq 30$ Gray. Meacham and colleagues observed a 1.8-fold increased risk of developing diabetes among childhood cancer survivors compared to sibling controls. ${ }^{2}$ In particular, high diabetes risks were observed after treatment with radiotherapy, total body irradiation, alkylating agents and treatment at ages $<4$ years. Among $\mathrm{HL}$ survivors, van Nimwegen and colleagues observed a 1.8-fold increased risk of diabetes after para-aortic radiotherapy with a prescribed dose of $\geq 36$ Gray compared to no para-aortic radiotherapy. ${ }^{4}$ So far, only one cohort study investigated diabetes risk after radiotherapy for testicular cancer. Haugnes and colleagues found a higher prevalence of diabetes after radiotherapy exposure (10.2\%, 38 diabetes patients) compared to surgery only $(4.0 \%, 8$ diabetes patients), but did not provide relative risk estimates. ${ }^{6}$ Although para-aortic radiotherapy for TC does not include the pancreatic tail and generally lower para-aortic doses are used compared to treatment of childhood cancer and $\mathrm{HL}$ patients, infradiaphragmatic radiotherapy was associated with an elevated diabetes risk in TC survivors. Somewhat surprisingly, patients treated with a para-aortic dose $\geq 33$ Gray did not have a higher diabetes risk than patients who received doses $\leq 26$ Gray. However, since only a small number of patients had doses $\geq 33$ Gray, our power to reliably establish an increased diabetes risk within this dose category was low.

Diabetes induced by infradiaphragmatic radiotherapy may result from direct damage to the pancreatic beta-cell Islets of
Langerhans $s^{1,2,4}$ or to the pancreatic microvasculature. ${ }^{21,22}$ A study in primates showed that pancreatic radiotherapy induced degranulation, vacuolisation, mitochondrial destruction and impaired insulin secretion shortly after treatment. ${ }^{23}$ Adjuvant radiotherapy has also been shown to reduce beta-cell function and insulin secretion capacity of the pancreas in 1-year gastric cancer survivors. ${ }^{22}$ Meacham and colleagues hypothesised that the radiotherapy effect is independent of obesity and therefore a result of beta-cell deficiency. ${ }^{2}$ Others found a marginally but not significantly larger diabetes risk among those with increased BMl. ${ }^{1,4}$ In our study, obesity at baseline was an independent risk factor for diabetes but did not modify radiotherapy-associated diabetes risk.

The pathway from para-aortic radiotherapy to diabetes could also be modified by subclinical hypogonadism after orchidectomy, which has been shown to increase the risk of developing a metabolic syndrome. ${ }^{24}$ Hypogonadism is associated with increased insulin insensitivity and diabetes risk. ${ }^{25,26}$ In the study of Haugnes and colleagues, a 2.3-fold increased odds for diabetes was observed after radiation compared to surgery only. ${ }^{6}$ Serum testosterone levels were lower after radiation compared to surgery only, but patients with orchidectomy only did not experience increased diabetes risk compared to general population controls. ${ }^{6}$ In our study the prevalence of hypogonadism was similar after radiation versus surgery (16\% after orchidectomy only and $18 \%$ after radiotherapy). Therefore, this pathway does not appear to play a major role in diabetes etiology.

We observed a fairly large number of incident diabetes cases $(N=161)$. Our risk estimates may underestimate the true risk, as we may have missed asymptomatic diabetes cases. As a booster dose was not recorded, we may also have underestimated the true dose to the pancreas in some patients, which could have attenuated our risk estimates for the association with prescribed radiation dose. Our study provides reliable risk estimates for the association of PAO-RT and diabetes risk as we had access to detailed treatment data, including relapse treatment.

Survivors of testicular cancer could be more health-conscious than their peers in the general population which can have influenced their lifestyle following testicular cancer treatment. Since diabetes in part depends on life-style, this may explain the unincreased risk of diabetes among irradiated patients compared to the general population. We did not find evidence for a more healthy lifestyle at diagnosis among irradiated and non-irradiated patients in the subcohort. Prevalence of smoking did not differ between these patients and smoking at diagnosis was not associated with diabetes risk. Although BMl above $30 \mathrm{~kg} / \mathrm{m}^{2}$ was associated with diabetes risk, prevalence of diabetes was similar in both groups. We found no evidence for confounding nor for modification of the radiation associated diabetes risk by $\mathrm{BMI}$, although imputation was necessary since for approximately $40 \%$ of the patients either weight or height was missing. A limitation of our general population comparisons is left-censoring in the analysis due to lack of reference data before 2002, resulting in exclusion of many person-years from analysis, mainly affecting patients treated in the distant past who were more frequently irradiated.

Although infradiaphragmatic radiotherapy is currently largely abandoned in treatment of stage I seminoma, while stage II seminoma patients with increased risk of metastatic disease still have an indication for radiotherapy, the association of previous para-aortic irradiation with diabetes risk is still very relevant for many long-term TC survivors. Radiotherapy techniques have improved over the past decades, with increasing use of CT planning since the late ' 90 s, which has resulted in more tailored abdominal RT fields. However, this did not necessarily decrease radiation field width in our study. In some cases, the amount of irradiated tissue near the renal hilum may even have increased over time. Currently, there are still no recommended constraints for the radiation dose to the pancreatic gland. We expect 
therefore that the change in the pancreatic volume included within the para-aortic radiation field over time was limited. In more recently irradiated patients diabetes risk may be lower due to the use of more precise intensity modulated radiotherapy techniques, exposing a lower proportion of the pancreas to a relatively high radiation dose. Nonetheless, this study suggests another reason why stage I seminoma patients may benefit from surveillance above undergoing PAO-RT, beyond the observation that the 5-year relapse rate of $4-6 \%$ for patients without prognostic risk factors under surveillance protocols ${ }^{27,28}$ is not that different from the 4\% 5-year relapse rate after abdominal RT. ${ }^{27,29}$

In conclusion, TC survivors treated with para-aortic irradiation have a 1.66-fold higher risk of diabetes compared to TC survivors treated with surgery only. Although diabetes risk is only moderately increased after para-aortic irradiation, and the relative risk in our study is lower compared to the risks observed in childhood cancer survivors, early detection and treatment of diabetes may prevent or alleviate late vascular damage and CVD. With the current improvements in radiation techniques the dose to organs at risk can be reduced, especially if the pancreas is defined as an organ at risk which is also important in radiation treatment of other malignancies. Unfortunately the optimal dose constraint cannot be derived from this study, although our study suggests to keep the dose to the pancreas below 26 Gray. This novel finding does not only apply to irradiated TC patients, but potentially also to patients with abdominal lymphoma, cervical cancer or stomach cancer who receive abdominal irradiation.

\section{ACKNOWLEDGEMENTS}

We would like to thank M.J. Nielen from the Netherlands Institute for Public Health Research for providing incidence data on diabetes for the general Dutch population. In addition, we thank T. Bootsma, M. Berkhof, S. Fase, and K. Kooijman (Netherlands Cancer Institute, Amsterdam, the Netherlands) for collecting data from the medical records and all participating general practitioners for completing questionnaires. This work was supported by the Dutch Cancer Society, grant number KWF-2011-5209.

\section{AUTHOR CONTRIBUTIONS}

Conception and design: H.J.G., M.S., F.E.v.L.

Provision of study materials or patients: B.M.P.A., A.W.v.d.B.-D., L.I., R.d.W., J.A.W., A. C.M.v.d.B., G.G., P.d.B., H.A.v.d.B., M.C.C.M.H., O.W.M.M., T.J.S., M.J.A., B.G.L.V., S.H., J.M. K., J.A.G., F.E.v.L.

Collection and assembly of data: H.J.G, M.S., A.v.d. B.-D., S.L., F.E.v.L.

Data analysis and interpretation: H.J.G., M.S., F.E.v.L., K.J.

Manuscript writing and final approval of manuscript: All authors.

Availability of data and materials: data were collected at NKI-AVL and are not publicly available.

\section{ADDITIONAL INFORMATION}

Supplementary information is available for this paper at https://doi.org/10.1038/ s41416-018-0248-x.

Competing interests: The authors declare no competing interests.

Ethics approval: The study protocol was submitted to the Institutional Review Board of the Netherlands Cancer Institute, which waived the requirement for individual patient consent. STROBE statement All data are presented in accordance with STROBE guidelines.

Note: This work is published under the standard license to publish agreement. After 12 months the work will become freely available and the license terms will switch to a Creative Commons Attribution 4.0 International (CC BY 4.0).

\section{REFERENCES}

1. de Vathaire, F. et al. Radiation dose to the pancreas and risk of diabetes mellitus in childhood cancer survivors: a retrospective cohort study. Lancet Oncol. 13, 1002-1010 (2012).

2. Meacham, L. R. et al. Diabetes mellitus in long-term survivors of childhood cancer. Increased risk associated with radiation therapy: a report for the childhood cancer survivor study. Arch. Intern. Med. 169, 1381-1388 (2009).

3. Meacham, L. R. et al. Cardiovascular risk factors in adult survivors of pediatric cancer-a report from the childhood cancer survivor study. Cancer Epidemiol. Biomark. Prev. 19, 170-181 (2010).

4. van Nimwegen, F. A. et al. Risk of diabetes mellitus in long-term survivors of Hodgkin lymphoma. J. Clin. Oncol. 32, 3257-3263 (2014).

5. Hauptmann, M. et al. Increased pancreatic cancer risk following radiotherapy for testicular cancer. Br. J. Cancer 115, 901-908 (2016).

6. Haugnes, H. S. et al. Cardiovascular risk factors and morbidity in long-term survivors of testicular cancer: a 20-year follow-up study. J. Clin. Oncol. 28, 4649-4657 (2010).

7. Stringhini, S. et al. Socioeconomic status and the $25 \times 25$ risk factors as determinants of premature mortality: a multicohort study and meta-analysis of 1.7 million men and women. Lancet 389, 1229-1237 (2017).

8. Grover, S. A., Paquet, S., Levinton, C., Coupal, L. \& Zowall, H. Estimating the benefits of modifying risk factors of cardiovascular disease: a comparison of primary vs secondary prevention. Arch. Intern. Med. 158, 655-662 (1998).

9. Conroy, R. M. et al. Estimation of ten-year risk of fatal cardiovascular disease in Europe: the SCORE project. Eur. Heart J. 24, 987-1003 (2003).

10. van den Belt-Dusebout, A. W. et al. Long-term risk of cardiovascular disease in 5year survivors of testicular cancer. J. Clin. Oncol. 24, 467-475 (2006).

11. van den Belt-Dusebout, A. W. et al. Treatment-specific risks of second malignancies and cardiovascular disease in 5-year survivors of testicular cancer. J. Clin. Oncol. 25, 4370-4378 (2007).

12. Barlow, W. E., Ichikawa, L., Rosner, D. \& Izumi, S. Analysis of case-cohort designs. J. Clin. Epidemiol. 52, 1165-1172 (1999).

13. van Leeuwen, F. E. et al. Second cancer risk following testicular cancer: a followup study of 1,909 patients. J. Clin. Oncol. 11, 415-424 (1993).

14. Zwaveling, A. \& Soebhag, R. Testicular tumors in the Netherlands. Cancer 55, 1612-1617 (1985).

15. Cohn-Cedermark, G., Stahl, O. \& Tandstad, T., Swenoteca. Surveillance vs. adjuvant therapy of clinical stage I testicular tumors - a review and the SWENOTECA experience. Andrology 3, 102-110 (2015).

16. Oliver, R. T. et al. Radiotherapy versus single-dose carboplatin in adjuvant treatment of stage I seminoma: a randomised trial. Lancet 366, 293-300 (2005).

17. Schmoll, H. J. et al. European consensus on diagnosis and treatment of germ cell cancer: a report of the European Germ Cell Cancer Consensus Group (EGCCCG). Ann. Oncol. 15, 1377-1399 (2004).

18. Rutten GEHM, D. G. W. et al. NHG-standaard diabetes mellitus type 2 ((derde herziening)). Huisarts. Wet. 56, 512-525 (2013).

19. Fine, J. A proportional hazards model for the subdistribution of a competing risk. J. Am. Stat. Assoc. 94, 496-509 (1999).

20. Rubin, D. B. Multiple Imputation for Nonresponse in Surveys (John Wiley \& Sons, New York, NY, 1987).

21. Oeffinger, K. C. \& Sklar, C. A. Abdominal radiation and diabetes: one more piece in the puzzle. Lancet Oncol. 13, 961-962 (2012).

22. Gemici, C. et al. Risk of endocrine pancreatic insufficiency in patients receiving adjuvant chemoradiation for resected gastric cancer. Radiother. Oncol. 107, 195-199 (2013).

23. Du Toit, D. F. et al. The effect of ionizing radiation on the primate pancreas: an endocrine and morphologic study. J. Surg. Oncol. 34, 43-52 (1987).

24. de Haas, E. C. et al. Early development of the metabolic syndrome after chemotherapy for testicular cancer. Annals of oncology: official journal of the European Society for. Med. Oncol. 24, 749-755 (2013).

25. Traish, A. M., Saad, F. \& Guay, A. The dark side of testosterone deficiency: II. Type 2 diabetes and insulin resistance. J. Androl. 30, 23-32 (2009).

26. Selvin, E. et al. Androgens and diabetes in men: results from the Third National Health and Nutrition Examination Survey (NHANES III). Diabetes Care 30, 234-238 (2007).

27. Tandstad, T. et al. Treatment of stage I seminoma, with one course of adjuvant carboplatin or surveillance, risk-adapted recommendations implementing patient autonomy: a report from the Swedish and Norwegian Testicular Cancer Group (SWENOTECA). Ann. Oncol. 27, 1299-1304 (2016).

28. Aparicio, J. et al. Risk-adapted management for patients with clinical stage I seminoma: the Second Spanish Germ Cell Cancer Cooperative Group study. J. Clin. Oncol. 23, 8717-8723 (2005).

29. Mead, G. M. et al. Randomized trials in 2466 patients with stage I seminoma: patterns of relapse and follow-up. J. Natl Cancer Inst. 103, 241-249 (2011). 